\title{
A 5-year review of malaria surveillance in Kuching, Sarawak
}

\author{
Haironi Y and Kamaliah MD \\ Dept of Community Med \& Public Health, Faculty of Medicine \& Health Sciences, Universiti Malaysia Sarawak
}

\begin{abstract}
Introduction: Malaria is endemic in Sarawak contributing to the highest incidence rate in Malaysia. Kuching division consists of three main areas, namely Kuching district, Lundu and Bau. This review aims to highlight the trends of malaria over the past five years in terms of demographic characteristics and vector control programmes and to identify factors that contribute to the difficulty in malaria control programme in this state. Materials and Methods: This is a descriptive review of malaria cases based on surveillance data compiled from 2000 to 2004. Data is analysed based on demographic background, malaria species, type of malaria infection and vector control activities between the three districts. Results: Lundu has the highest average 5-year incidence rate per 10,000 population of 121.97 as compared to the national figure of 4.10. Malaria is more prevalent in males (14.3\%) than females (5.7\%). Among the race, Dayaks had the highest prevalence rate (66.5\%). Peak age affected is between 15 to 40 years. The predominant species was Plasmodium vivax (65.4\%) followed by Plasmodium falciparum (32.8\%). For occupation, the majority of cases were among villagers (55.2\%) followed by foreign workers (19.0\%). Cases were detected mainly by passive case detection (82.7\%) and blood film for malarial parasites was positive in $2.67 \%$. For malaria control activities, focal spraying were successfully done in $94.4 \%$ of the structures, regular spraying in $89.5 \%$ structures, impregnated bednet distribution among $38.2 \%$ population and mass drug administration distributed among $\mathbf{8 0 . 7 \%}$ of the population. Conclusion: Results analysis has shown that the incidence rate of malaria in Kuching division is very high, particularly in Lundu district. Although activities conducted by the malaria control programme is sufficient, it has not been able to successfully reduce the incidence of malaria in this region. Problems identified include difficulty in accessing the affected areas, movements of illegal immigrants, financial constraints and lack of training of field staff involved in malaria control. Recommendations include integrating malaria control measures with cross border agents, community participation, aggressive surveillance programmes and improvement of vector control.
\end{abstract}

KEYWORDS: Review, malaria, prevalence 